\title{
CALCIUM HEXAFERRITE POWDERS PREPARED BY A MICROWAVE- INDUCED SOL-GEL COMBUSTION ROUTE AND STUDY OF SOME OF THEIR PROPERTIES
}

\author{
${ }^{1}$ A. D. Deshpande; ${ }^{2}$ V. M. Nanoti, ${ }^{3 K}$. G. Rewatkar \\ 1,2Dept. of Applied Physics, Priyadarshini College of Engineering, Nagpur \\ Dept. of Physics, Dr. Ambedkar College, Nagpur-10
}

\begin{abstract}
Powders of calcium hexaferrites doped with Zirconium - Cobalt $\mathrm{CaFe}_{11}(\mathrm{ZrCo}) \mathrm{O}_{19}$ and $\mathrm{CaFe}_{10}(\mathrm{ZrCo})_{2} \mathrm{O}_{19}$ have been synthesized. The nitrates are used as oxidants accompanied with urea as a fuel. Conventional Sol-gel technique is improved by replacing conventional heating furnace by a microwave source, hence the method is named as 'The Microwave Induced sol-gel combustion route' [1,2]. Structural analysis of the synthesized powders have been carried out using X-ray diffraction spectroscopy (XRD)\& Morhological study is done using Transmission electron microscopy (TEM). Values of Lattice parameters $(a, c)$ confirms the formation of Hexaferrite unit cell [3]. TEM imaging demonstrate the formation of nanosize particles. Variatoin of loss tangent $(\tan \delta)$ is studied as a function of frequency using precision impendence Analyser. Value of DC electrical resistivity $(\rho)$ is also determined for the samples. Response to the external applied magnetic field is studied using Vibrating sample magnetometer (VSM), which show the moderate values of saturation magnetization (Ms), Remenace (Mr) and Coercivity (Hc). These nano sized materials with high resistivity are reported to be useful specially for the many Microwave absorbing applications. Also the reduction of particle size of hexaferrites to nanorange helps to improve magnetic and electric properties mentioned earlier.
\end{abstract}

\section{Introduction}

In modern technoscientific era, the industrial application of nano materials has grabbed a principal importance owing to their improved electric and magnetic characteristics. Hexagonal ferrites especially Mtype ferrites have been proved to be the promising candidates for nano materials due to their ease of applicability in high density recording media, microwave absorption devices, magneto-optic recording media, etc. Ferrite is a very important class of magnetic oxides which contains 
magnetic ions arranged in such a manner that it produces spontaneous magnetization while maintaining good dielectric properties. The present module of research aims to develop a systematic method which facilitates the production of ultra fine hexagonal ferrite particles especially in nano scale and to find possible ways of improving their behavioral characteristics. Many efforts have been devoted to control structural Mtype magnetoplumbites merely by the substitution of trivalent or coupled divalent \& tetravalent cations into the mother base of these ferrites. Substituted magnetoplumbites with $\mathrm{Ca}^{2+}$ for $\mathrm{Ba}^{2+}$, trivalent metallic ion for $\mathrm{Fe}^{3+}$ or a combination of divalent and tetravalent ions for $\mathrm{Fe}^{3+}$ have been reported[4]. The calcium hexaferrits [5] attracted the attention as they have been less studied and have magnetic properties comparable to BaM or SrM.

There are many procedures for synthesis. The known sol-gel technique is reported to have many advantages for structural, electrical and magnetic parameters over old techniques like solid state method, co-precipitation, Hydro-thermal synthesis, Glass crystallization, etc. [6]. The magnetic particles produced by conventional methods are reported to be larger than those produced by this technique. This technique is improved by replacing conventional heating furnace by scientific microwave source of specific frequency, hence the name 'The Microwave Induced sol-gel combustion route'.

In this module, samples synthesized by Microwave Induced sol-gel combustion route with varying concentrations of dopants are described and their structural, electric and the magnetic properties are discussed.

\section{Experimental}

The synthesis of powders of $\mathrm{CaFe}_{11}\left(\mathrm{ZrCo} \mathrm{O}_{19}\right.$ and $\mathrm{CaFe}_{10}\left(\mathrm{ZrCo}_{2} \mathrm{O}_{19}\right.$ is done using the sol-gel route [7]. The reactive oxidants such as $\mathrm{Ca}\left(\mathrm{NO}_{3}\right)_{2}$, Fe $\left(\mathrm{NO}_{3}\right)_{2 .} .9 \mathrm{H}_{2} \mathrm{O}, \mathrm{Co}\left(\mathrm{NO}_{3}\right)_{2} .6 \mathrm{H}_{2} \mathrm{O}, \mathrm{ZrO}\left(\mathrm{NO}_{3}\right)_{2} . \mathrm{H}_{2} \mathrm{O}$ were dissolved into an unionized distilled water at the temperature of $50^{\circ} \mathrm{C}$ for $15-20 \mathrm{~min}$, urea 
is used as fuel which gives requisite energy to initiate exothermic reaction. The gel produced is then kept for an hour in the room temperature and then it is heated in the digitally controlled microwave oven of $2.54 \mathrm{GHz}$ for $15-20 \mathrm{~min}$. The gel get burnt and finally gets converted in homogeneous powder. The sample is then sintered by giving sporadic moderate heat treatments for further few minutes with further grinding. The samples so produced are then kept in humidity free atmosphere in air tight compartment. Pellets of diameter $15 \mathrm{~mm}$ are formed using hydraulic press. Polyvinyl acetate (PVA) is used as binder for pellets and the sintering is done at $400^{\circ} \mathrm{C}$ for 2 hours by sequentially increasing and decreasing temperature at the moderate rate of $4^{\circ} \mathrm{C} / \mathrm{min}$.

\section{Result and discussion}

Table 1 gives the values of the Structural, Electric and Magnetic properties of the samples.

\subsection{Structural Properties}

The diffraction patterns of samples are taken with Philips X'pert Diffractometer, using $\mathrm{Cu} \mathrm{K} \alpha$ radiation with wavelength $\lambda=1.542$ A.U.

\section{Fig 1 . X-ray powder diffraction patterns of samples}

(a)

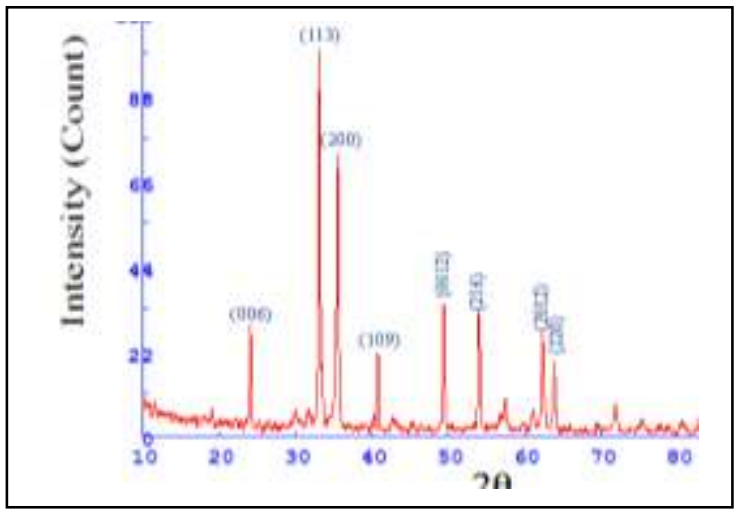

(b)

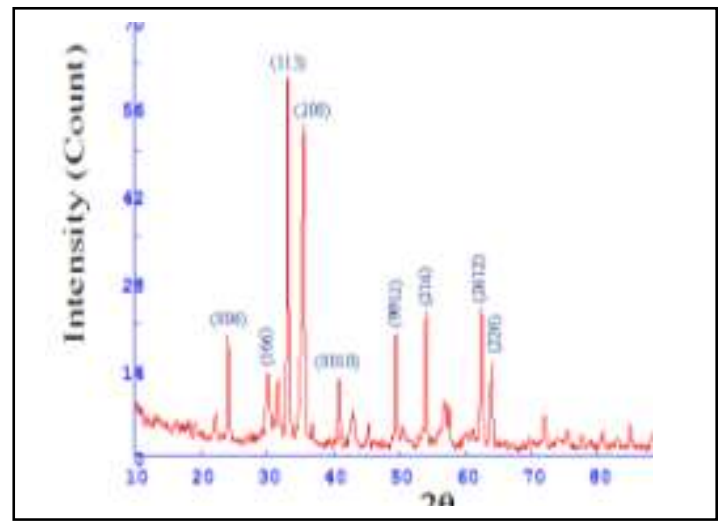

Fig 1 (a-b) shows the intensity graphs for samples $\mathrm{CaFe}_{11}\left(\mathrm{ZrCo}_{\mathrm{O}} \mathrm{O}_{19}\right.$ and $\mathrm{CaFe}_{10}(\mathrm{ZrCo})_{2} \mathrm{O}_{19}$ respectively. By comparing the diffraction patterns with JCPDS standards, using $2 \theta$ values, observed d-values and intensity 
variations, d-values are recalculated and (hkl) planes are finalized. Values of lattice parameters confirms the formation of single phase hexagonal ferrites. Lattice parameters $a$ and $c$ are found to be $5.8256(\AA)$. and $22.1244(\AA)$. respectively for $\mathrm{CaFe}_{10}\left(\mathrm{ZrCo}_{2} \mathrm{O}_{19}\right.$ and 5.8234 ( $\left.\mathrm{A}\right)$. and 22.1286 (Å). for $\mathrm{CaFe}_{10}\left(\mathrm{ZrCo}_{2} \mathrm{O}_{19}\right.$ respectively. The space group of samples is observed to be $P 63 / \mathrm{mmc}$.

Nanosize confirmation is done by TEM analysis using Philips CM200, Fig 2 (a-b) shows the TEM images of the samples. The particle size is found to on an average 100nm. The grains are found to have sharp edges.

\section{$\mathrm{CaFe}_{10}(\mathrm{ZrCo})_{2} \mathrm{O}_{19}$}

(a)

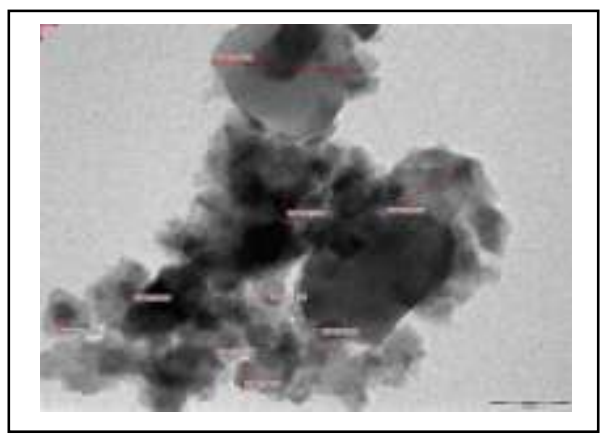

\section{$\mathrm{CaFe}_{11}\left(\mathrm{ZrCo} \mathrm{O}_{19}\right.$}

(b)

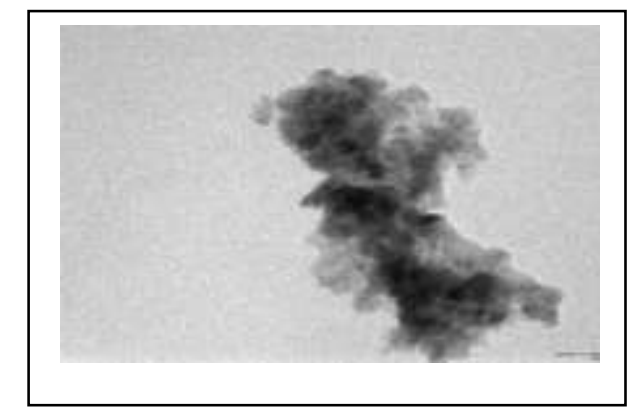

Fig. 2 (a-b) Transmission electron micrographs of the samples

\subsection{Electric \& Dielectric Properties}

The variation of Resistivity, loss tangent(tan $\delta)$, with respect to frequency, temperature and composition are studied using precision impendence Analyser 6500B, Waynekerr Electronics. For controlled temperature variation, the sample is kept in a digitally controlled furnace. Four probe method is used to measure dielectric properties of the samples in palletized form.

From Table 1 it is observed that resistivity at room temperature decreases from, $1.2292 * 10^{7} \Omega-\mathrm{m}$ to $1.7718^{*} 10^{6} \Omega-\mathrm{m}$ with increase in 
substitution of cations. Electric and dielectric properties of the hexaferrites are explained on the basis Verwey's hopping mechanism [8] The dielectric loss (tand) gives energy loss within the material during conduction of electrons. Variation of dielectric loss (tan $\delta$ ) with frequency is shown in Fig 3 at constant temperature. The value of loss tangent is observed to decrease with increasing concentration of substitution. Lagging of the polarization with applied ac electric field is the main cause of the dielectric loss [9]. In low frequency region more energy is required for the electron exchange between $\mathrm{Fe}^{3+}$ and $\mathrm{Fe}^{2+}$ ions due to which loss is higher. But at higher frequencies lesser energy is required for the exchange and therefore dielectric loss decreases rapidly.

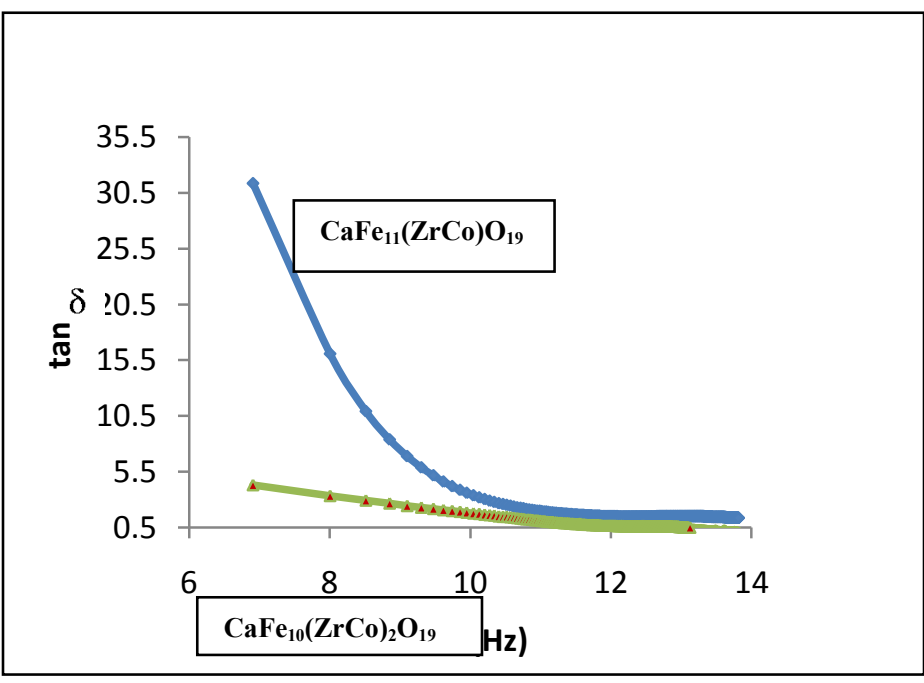

Fig 3 : Variation of dielectric loss $(\tan \delta)$ with frequency for $\mathrm{CaFe}_{11}(\mathrm{ZrCo})_{19}$ and $\mathrm{CaFe}_{10}\left(\mathrm{ZrCo}_{2} \mathrm{O}_{19}\right.$

\subsection{Magnetic Properties}

Fig. 4 shows hysteresis curves for the sample depicting magnetic behavior of the sample in the presence of external magnetic field.

Values of saturation magnetization $\mathrm{Ms}$, remnant magnetization $\mathrm{Mr}$ and coercivity Hc found to decreases with the substitution of cations as mentioned in Table 1. Decrease in the values of Ms, Mr with increase in values of substitution is due to the substitution of diamagnetic ions not 
only into the spin-down sublattices $4 \mathrm{f}_{1}$ of $\mathrm{Fe}^{3+}$ ions but also into other octahedral spin-up sublattices $12 \mathrm{k}, 2 \mathrm{a}, 2 \mathrm{~b}$ of $\mathrm{Fe}^{3+}$ ions. Decrease in the value of coercivit Hc with increase in the value of substitution is due to lowering of magneto crystalline anisotropy[10] .

Also the area under hysteresis curves decreases with increase in substitution level which indicates that the ferrite become soft with increasing degree of substitution.

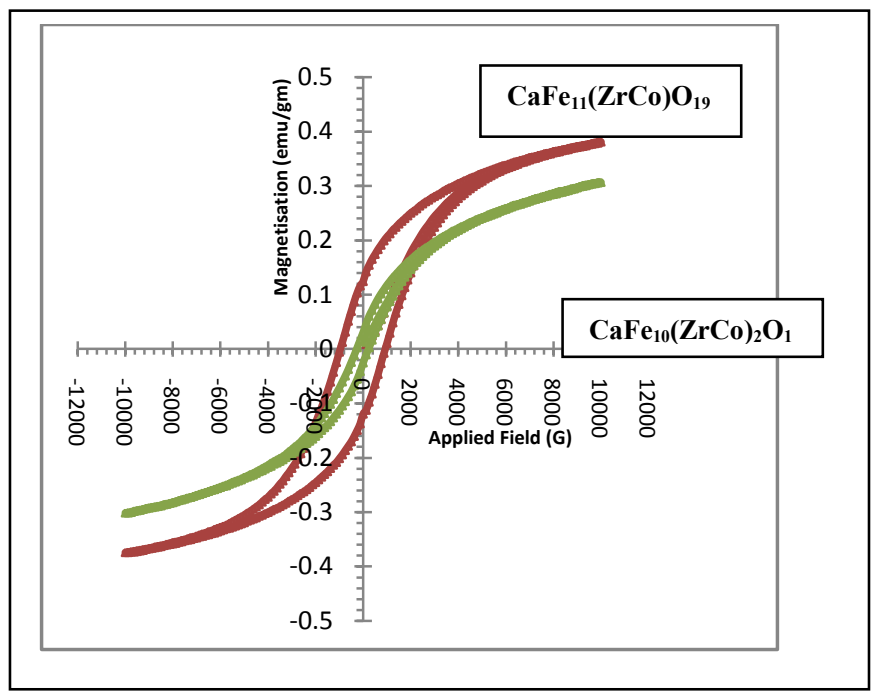

Fig. 3 Hysteresis curves for the samples $\mathrm{CaFe}_{10}(\mathrm{ZrCo})_{2} \mathrm{O}_{19}$ and $\mathrm{CaFe}_{11}\left(\mathrm{ZrCo} \mathrm{O}_{19}\right.$

Table 1 : Lattice parameters ( a and c), Bulk Density $\left(D_{B}\right)$, X-ray Density $\left(D_{\mathrm{x}}\right), \%$ Porosity (P\%), Values of saturation magnetization $\mathbf{M}_{\mathbf{s}}$, remnant magnetization $\mathbf{M}_{\mathbf{r}}$ and coercivity $\mathbf{H}_{c}$

\begin{tabular}{|c|c|c|c|c|c|c|c|}
\hline Samples & $\mathbf{a}(\AA)$ & $\mathbf{c}(\AA)$ & $\begin{array}{c}\mathbf{\rho} \text { at 373K } \\
(\Omega-\mathbf{m})\end{array}$ & $\begin{array}{c}\text { tan } \mathbf{~ a t} \\
\mathbf{1 ~ k H z}\end{array}$ & $\begin{array}{c}\mathbf{M s} \\
(\mathbf{e m u} / \mathbf{g m})\end{array}$ & $\begin{array}{c}\mathbf{M r} \\
(\mathbf{e m u} / \mathbf{g m})\end{array}$ & $\mathbf{H c}(\mathbf{G})$ \\
\hline $\mathrm{CaFe}_{11}(\mathrm{ZrCo}) \mathrm{O}_{19}$ & 5.8256 & 22.124 & $1.2292^{*} 10^{7}$ & 2.6238 & 0.3805 & 0.1245 & 875 \\
\hline $\mathrm{CaFe}_{10}(\mathrm{ZrCo})_{2} \mathrm{O}_{19}$ & 5.8234 & 22.128 & $1.7718^{*} 10^{6}$ & 0.1314 & 0.3055 & 0.0305 & 250 \\
\hline
\end{tabular}

\section{Conclusion}

The new combustion route using Microwave found to be successful for the synthesis of nanosized $\mathrm{Co}^{2+}-\mathrm{Zr}^{4+}$ substituted calcium hexaferrites with added advantage of sol-gel method which gives the nanosized hexaferrite 
particles. The X-ray diffraction studies confirm the formation of hexaferrites and the a \& c values of the sample supports this confirmation. From TEM data, particle size is found to be in nano range. Dielectric losses are observed to decrease with increase in frequency. With high values of electrical resistivity and low values for dielectric loss tangent, the synthesized calcium hexaferrites dopped with $\mathrm{Zr}^{4+}$ and $\mathrm{Co}^{2+}$ can prove to be a promising material in the microwave absorbing, EMI shielding applications. Hysteresis curves of the samples depicts the moderate values of coercivity and large values of saturation magnetization. The variation in the values of magnetic parameters shows the possibility of tailoring of the magnetic properties according to the requirement for different utilities $[8,11]$. The average particle size will determine the background noise in the recording media. Nano size of the particles helps to reduce the noise produced in magnetic data storage devices which occur due to displacement of domain boundaries[2]. Thus the reduction of particle size of hexaferrites to nanorange helps to improve magnetic and electric properties.

\section{References}

G.B. Teh, N. Swaminathan, and D.A. Jefferson; (2007). Materials of Chemistry and Physics 105, 253-259

Sharad N. Sable, Kishor G. Rewatkar, Vivek M. Nanoti; (2009). Material science and Engineering-B, Article in Press.

X. Battle, M. Garcia del Muro, J. Tejada, H. Pfeiffer, P. G*ornert, and E. Sinn, (1994). IEEE Trans. Magn. 30 (2) 708-710.

K. Mallick, P. Shepherd, and R.J. Green, (2007). J. Eur. Ceram. Soc. 27, 2045-2052.

D. K. Kulkarni and C S Prakash, (1993). Bull. Mat Sci., 35-39

M. Edrissi, and R. Norouzbeigi, (2007). Material Science 23 (4). 
Simon Thompson, Neil J. Shirtcliffe, Eoin S., O’Keefe, Steve Appleton, and Carole C. Perry; (2005). Journal of Magnetic and Magnetic Materials, 292, 100-107

D.A. Jefferson, J.M. Thomas, G.R. Millward, K. Tsuno, A. Harriman, and R.D. Brydson, (1986). Nature 323, 428.

E.J.W. Verwey, and J.H. De Boer, (1936). Rec. Trans. Chem. Des. Pays. Bas 55: 531

V.V. Soman, V.M. Nanoti, and D.K. Kulkarni, (2013). Cerm. Inter. 39: 5713-5723

T. Gonzalez-Carren, (2000). M.P. Morales, C,J. Serna, Materials Letters $43,97$. 